\title{
Relationship between Diversity and Stability of a Karst Plant Community
}

\author{
Yang Wang ${ }^{1}$, Jin Chen $^{1}$, Limin Zhang ${ }^{1}$, Ling Feng ${ }^{1}$, Lingbin Yan ${ }^{1}$, Fangbing Li $^{1}$, Xiangwei \\ $\mathrm{Zhao}^{1}$, and Lifei $\mathrm{Yu}^{1}$ \\ ${ }^{1}$ Guizhou University
}

January 13, 2022

\begin{abstract}
The relationships among species diversity, functional diversity, functional redundancy, and community stability are central to community and ecosystem ecology. This paper examines plant communities at different stages of vegetation restoration in the Guizhou karst plateau to study the relationship among functional diversity, functional redundancy, and stability of plant communities. The most important results include the following. (1) Species diversity (SD), functional redundancy (FR), and stability (STB) gradually increased with restoration, and there were significant differences among the different stages; functional diversity (FD) increased at first and then decreased, and reached the highest level at the tree irrigation stage. (2) Plant height $(\mathrm{PLH})$ and specific leaf area (SLA) were functional traits that affected the diversity and stability of the plant community, and PLH was positively correlated with plant community diversity and stability, while SLA was negatively correlated with plant community diversity and stability. (3) During the community recovery, FD and FR interacted to maintain stability. In the early and late stages of recovery, the effect of functional redundancy on stability was greater than that of functional diversity, but it was the opposite in the middle stages. (4) The tree irrigation stage is the likely point at which the species diversity of plant communities in karst areas reached saturation, and the growth rate of functional redundancy after species diversity saturation was greater than that before saturation.
\end{abstract}

\section{Hosted file}

Relationship between Diversity and Stability of a Karst Plant Community.docx available at https://authorea.com/users/455123/articles/552598-relationship-between-diversity-andstability-of-a-karst-plant-community 